\title{
Schatten class Toeplitz operators on weighted Bergman spaces of tube domains over symmetric cones
}

\author{
Benoît F. Sehba* (i)
}

*Correspondence: bfsehba@ug.edu.gh Department of Mathematics, University of Ghana, P. O. Box LG 62, Legon, Accra, Ghana

\section{Abstract}

We prove some characterizations of Schatten class of Toeplitz operators on Bergman spaces of tube domains over symmetric cones for small exponents. Our results are applied to obtain Schatten class characterization of some Cesàro-type operators.

Keywords: Bergman space, Besov space, Cesàro-type operator, Toeplitz operator, Schatten class, Symmetric cone

Mathematics Subject Classification: Primary 32A10, 32A38; Secondary 47B32, $32 \mathrm{M} 15$

\section{Introduction}

All over the text, $\Omega$ will denote an irreducible symmetric cone in $\mathbb{R}^{n}$, and $\mathcal{D}=\mathbb{R}^{n}+i \Omega$ the tube domain over $\Omega$. As in [10] we denote by $r$ the rank of the cone $\Omega$ and by $\Delta$ the associated determinant function in $\mathbb{R}^{n}$. We recall that for $n \geq 3$, when $r=2$, as example of symmetric cones, we have the Lorentz cone $\Lambda_{n}$ which is defined by

$$
\Lambda_{n}=\left\{\left(y_{1}, \ldots, y_{n}\right) \in \mathbb{R}^{n}: y_{1}^{2}-\cdots-y_{n}^{2}>0, \quad y_{1}>0\right\} ;
$$

its associated determinant function is given by the Lorentz form

$$
\Delta(y)=y_{1}^{2}-\cdots-y_{n}^{2} .
$$

As usual, we denote by $\mathcal{H}(\mathcal{D})$ the space of holomorphic functions on $\mathcal{D}$.

For $1 \leq p<\infty$ and $v \in \mathbb{R}$, we write $\mathrm{d} V_{v}(x+i y)=\Delta^{v-\frac{n}{r}}(y) \mathrm{d} x \mathrm{~d} y$. Given $1 \leq p<\infty$ and $v \in \mathbb{R}, L_{v}^{p}(\mathcal{D})=L^{p}\left(\mathcal{D}, \mathrm{d} V_{v}\right)$ denotes the space of all functions $f$ satisfying the condition

$$
\|f\|_{p, v}=\|f\|_{L_{v}^{p}(\mathcal{D})}:=\left(\int_{\mathcal{D}}|f(x+i y)|^{p} \Delta^{\nu-\frac{n}{r}}(y) \mathrm{d} x \mathrm{~d} y\right)^{1 / p}<\infty .
$$

The weighted Bergman space $A_{v}^{p}(\mathcal{D})$ is the closed subspace of $L_{v}^{p}(\mathcal{D})$ consisting of holomorphic functions in $\mathcal{D}$. Following [9], this space is not trivial (i.e., $A_{v}^{p}(\mathcal{D}) \neq\{0\}$ ) only if $v>\frac{n}{r}-1$. The weighted Bergman projection $P_{v}$ is the orthogonal projection of the

(c) The Author(s) 2018. This article is distributed under the terms of the Creative Commons Attribution 4.0 International License (http://creativecommons.org/licenses/by/4.0/), which permits unrestricted use, distribution, and reproduction in any medium provided you give appropriate credit to the original author(s) and the source, provide a link to the Creative Commons license, and indicate if changes were made. 
Hilbert space $L_{v}^{2}(\mathcal{D})$ onto its closed subspace $A_{v}^{2}(\mathcal{D})$. It is well known that $P_{v}$ is an integral operator given by

$$
P_{v} f(z)=\int_{\mathcal{D}} K_{v}(z, w) f(w) \mathrm{d} V_{\nu}(w),
$$

where $K_{v}(z, w)=c_{v} \Delta^{-\left(v+\frac{n}{r}\right)}((z-\bar{w}) / i)$ is the weighted Bergman kernel, i.e., the reproducing kernel of $A_{v}^{2}(\mathcal{D})$ (see [10]). Here, we use the notation $\mathrm{d} V_{v}(w):=\Delta^{v-\frac{n}{r}}(v) \mathrm{d} u \mathrm{~d} v$, where $w=u+i v$ is an element of $\mathcal{D}$. The unweighted case corresponds to $v=\frac{n}{r}$.

For $\mu$ a positive Borel measure on $\mathcal{D}$, the Toeplitz operator $T_{\mu}$ is the operator defined for functions $f$ with compact support by

$$
T_{\mu} f(z):=\int_{\mathcal{D}} K_{\nu}(z, w) f(w) \mathrm{d} \mu(w),
$$

where $K_{v}$ is the weighted Bergman kernel.

Schatten class $\mathcal{S}_{p}(0<p \leq \infty)$ criteria of the Toeplitz operators have been obtained by several authors on bounded domains of $\mathbb{C}^{n}$ (see $[1,8,12,21,22]$ and the references therein). For unbounded domains, Schatten classes have been also characterized in Fock spaces by several authors (see for example [11, 14] and the references therein). In [13], we extended these results for $1 \leq p \leq \infty$ to weighted Bergman spaces of tube domains over symmetric cones. To be more precise, let us introduce more notations.

For $\delta>0$, we denote by

$$
B_{\delta}(z)=\{w \in \mathcal{D}: d(z, w)<\delta\}
$$

the Bergman ball centered at $z$ with radius $\delta, d$ is the Bergman distance on $\mathcal{D}$. For $v>\frac{n}{r}-1$ and $w \in \mathcal{D}$, the normalized reproducing kernel of $A_{v}^{2}(\mathcal{D})$ at $w$ is given by

$$
k_{v}(\cdot, w)=\frac{K_{v}(\cdot, w)}{\left\|K_{v}(\cdot, w)\right\|_{2, v}}=\Delta^{-v-\frac{n}{r}}\left(\frac{\cdot-\bar{w}}{i}\right) \Delta^{\frac{1}{2}\left(v+\frac{n}{r}\right)}(\Im w) .
$$

Let $\mu$ be a positive measure on $\mathcal{D}$. For $w \in \mathcal{D}$, we define

$$
\tilde{\mu}(w):=\int_{\mathcal{D}}\left|k_{v}(z, w)\right|^{2} \mathrm{~d} \mu(z) .
$$

The function $\tilde{\mu}$ is the Berezin transform of the measure $\mu$. For $z \in \mathcal{D}$ and $\delta \in(0,1)$, we define the average of the positive measure $\mu$ at $z$ by

$$
\hat{\mu}_{\delta}(z)=\frac{\mu\left(B_{\delta}(z)\right)}{V_{v}\left(B_{\delta}(z)\right)} .
$$

Consider the measure $\mathrm{d} \lambda(z)=\Delta^{-2 n / r}(\Im z) \mathrm{d} V(z)$. The following was obtained in [13].

Theorem 1.1 Let $\mu$ be a positive Borel measure on $\mathcal{D}$, and $v>\frac{n}{r}-1$. Then for $p \geq 1$, the following assertions are equivalent:

(i) The Toeplitz operator $T_{\mu}$ belongs to the Schatten class $\mathcal{S}_{p}\left(A_{v}^{2}(\mathcal{D})\right)$. 
(ii) For any $\delta$-lattice $(\delta \in(0,1))\left\{\zeta_{j}\right\}_{j \in \mathbb{N}}$ in the Bergman metric of $\mathcal{D}$, the sequence $\left\{\hat{\mu}_{\delta}\left(\zeta_{j}\right)\right\}$ belongs to $l^{p}$.

(iii) For any $\beta \in(0,1)$, the function $z \mapsto \hat{\mu}_{\beta}(z)$ belongs to $L^{p}(\mathcal{D}, \mathrm{d} \lambda)$, with $\mathrm{d} \lambda$ the invariant measure on $\mathcal{D}$.

(iv) $\tilde{\mu} \in L^{p}(\mathcal{D}, \mathrm{d} \lambda)$.

Our first concern in this note is for the extension of the above result to the range $0<p<1$. We prove that the equivalences (i) $\Leftrightarrow$ (ii) $\Leftrightarrow$ (ii) still hold for $\frac{2\left(\frac{n}{r}-1\right)}{v+\frac{n}{r}}<p<1$. This cut-off is due to integrability conditions of the determinant function. The equivalence with the last assertion in the above result still also holds if we restrict to $\frac{2 \frac{n}{r}-1}{v+\frac{n}{r}}<p<1$. The last cut-off point is also due to integrability conditions of the determinant function and one can prove that it is sharp. Our result is then as follows.

Theorem 1.2 Let $\mu$ be a positive Borel measure on $\mathcal{D}$, and $v>\frac{n}{r}-1$. Then for $\frac{\frac{n}{r}-1}{v+\frac{n}{r}}<p<1$. the following assertions are equivalent:

(i) The Toeplitz operator $T_{\mu}$ belongs to the Schatten class $\mathcal{S}_{p}\left(A_{v}^{2}(\mathcal{D})\right)$.

(ii) If $\frac{2\left(\frac{n}{r}-1\right)}{v+\frac{n}{r}}<p<1$, then for any $\delta$-lattice $(\delta \in(0,1))\left\{\zeta_{j}\right\}_{j \in \mathbb{N}}$ in the Bergman metric of $\mathcal{D}$, the sequence $\left\{\hat{\mu}_{\delta}\left(\zeta_{j}\right)\right\}$ belongs to $l^{p}$.

(iii) For any $\beta \in(0,1)$, the function $z \mapsto \hat{\mu}_{\beta}(z)$ belongs to $L^{p}(\mathcal{D}, \mathrm{d} \lambda)$.

Moreover, if $p>\frac{2 \frac{n}{r}-1}{v+\frac{n}{r}}$, then the above assertions are equivalent to the following

(iv) $\tilde{\mu} \in L^{p}(\mathcal{D}, \mathrm{d} \lambda)$.

We note that in the above result, all the relevant norms are equivalent. We also have that the implication (iii) $\Rightarrow$ (i) actually holds for the full range $0<p \leq 1$.

The main difficulty in the proof of the above theorem is the implication (i) $\Rightarrow$ (ii). The idea in [22] is to replace the measure $\mu$ by a measure supported on a disjoint union of Bergman balls, then split the associated Toeplitz operator into its diagonal and off-diagonal parts. It is not hard to prove that the Schatten norm of the diagonal part dominates the $l^{p}$-norm of the sequence $\left\{\hat{\mu}_{\delta}\left(\zeta_{j}\right)\right\}$. The difficulty is to prove that the latter norm dominates (up to a pretty small constant) the Schatten norm of the off-diagonal operator. A part of the techniques in [22] uses the fact that the unit ball is bounded, and so it cannot be used in our setting. We overcome this difficulty by using a technical lemma originally due to Békollé and Temgoua [7]. Considered even in the unit ball, our contribution heavily simplifies the proof of Zhu in [22].

We are also interested here in some other possible equivalent characterizations of Schatten class Toeplitz operators. For this, we denote by $\square_{z}$ the natural extension to $\mathbb{C}^{n}=\mathbb{R}^{n}+i \mathbb{R}^{n}$ of the wave operator $\square_{x}$ on the cone:

$$
\square_{z}=\Delta\left(\frac{1}{i} \frac{\partial}{\partial z}\right)
$$

which is the differential operator of degree $r$ defined by the equality: 


$$
\square_{z}\left[\mathrm{e}^{i(z \mid \xi)}\right]=\Delta(\xi) \mathrm{e}^{i(z \mid \xi)}, \quad z \in \mathbb{C}^{n}, \xi \in \mathbb{R}^{n} .
$$

We recall (see [5]) that $\square_{z}$ acts on the Bergman kernel as follows:

$$
\square_{z} K_{v}(z, w)=C_{v} K_{v+1}(z, w) .
$$

Let $m$ be a positive integer. For simplicity, we use the following notation for higher order derivatives of the Bergman kernel,

$$
K_{z}^{v, m}(w):=\square_{z}^{m} K_{v}(z, w)
$$

and

$$
k_{z}^{\nu, m}(w):=\frac{K_{z}^{\nu, m}(w)}{\left\|K_{z}^{\nu, m}(z, \cdot)\right\|_{2, v}}=C_{\nu, m} \Delta^{-\left(v+m+\frac{n}{r}\right)}\left(\frac{z-\bar{w}}{i}\right) \Delta^{\frac{1}{2}\left(v+2 m+\frac{n}{r}\right)}(\Im w) .
$$

Define the quantity

$$
\tilde{\mu}^{m}(z):=\left\langle T_{\mu} k_{z}^{v, m}, k_{z}^{v, m}\right\rangle_{\nu}=\int_{\mathcal{D}}\left|k_{z}^{v, m}(w)\right|^{2} \mathrm{~d} \mu(w) .
$$

We also have the following equivalent characterization.

Theorem 1.3 Let $v>\frac{n}{r}-1$, and $\frac{\frac{n}{r}-1}{v+\frac{n}{r}} \leq p<\infty$. Assume $\mu$ is a positive measure on $\mathcal{D}$. Then the following assertions are equivalent.

(a) The Toeplitz operator $T_{\mu}$ belongs to the Schatten class $\mathcal{S}_{p}\left(A_{v}^{2}(\mathcal{D})\right)$.

(b) For every integer $m \geq 0$ with $p>\frac{2 \frac{n}{r}-1}{v+\frac{n}{r}+2 m}$, we have $\tilde{\mu}^{m} \in L^{p}(\mathcal{D}, \mathrm{d} \lambda)$.

(c) For some integer $m \geq 0$ with $p>\frac{2 \frac{n}{r}-1}{v+\frac{n}{r}+2 m}$, we have $\tilde{\mu}^{m} \in L^{p}(\mathcal{D}, \mathrm{d} \lambda)$.

Again the condition $p \geq \max \left\{\frac{\frac{n}{r}-1}{v+\frac{n}{r}}, \frac{2 \frac{n}{r}-1}{v+\frac{n}{r}+2 m}\right\}$ is due to integrability conditions of the determinant function. We note that when $m=0$, the result corresponds to the equivalence (i) $\Leftrightarrow$ (iv) in Theorem 1.2. We also observe that the relevant norms in the above result are equivalent.

For the proof of Theorem 1.3, we derive the necessary condition for $1 \leq p<\infty$ and the sufficient condition for $0<p<1$ from a more general result for any positive operator. The proof of the other parts essentially uses the properties of Bergman balls and the $\delta$-lattices. We also refer to $[15,17]$ for this type of results.

We are essentially motivated here by the idea of extending the results in [13] for Toeplitz operators $T_{\mu}$ in Schatten classes $\mathcal{S}_{p}\left(A_{v}^{2}(\mathcal{D})\right)$ for $p \geq 1$, to the case $0<p<1$, and settling the problem of the characterization of Schatten class $\mathcal{S}_{p}\left(A_{v}^{2}(\mathcal{D})\right.$ ) for $1 \leq p<2$ for the Cesàro-type operator introduced in [13].

The paper is organized as follows: In the next section, we present some useful tools and results needed in the proofs of the above results. The proof of Theorem 1.2 is given in Sect. 3. In Sect. 4, we provide characterization of Schatten class for general positive operators. We prove Theorem 1.3 in Sect. 5. In the last section, we apply our results to extend to the range $1 \leq p<2$, the characterization of Schatten class $\mathcal{S}_{p}\left(A_{v}^{2}(\mathcal{D})\right.$ ) for the Cesàro-type operator obtained in [13]. 
As usual, given two positive quantities $A$ and $B$, the notation $A \lesssim B$ (resp. $A \gtrsim B$ ) means that there is an absolute positive constant $C$ such that $A \leq C B$ (resp. $A \geq C B$ ). When $A \lesssim B$ and $B \lesssim A$, we write $A \approx B$ and say $A$ and $B$ are equivalent. Finally, throughout the text, $C, C_{k}$, $C_{k, j}$ will denote positive constants depending only on the displayed parameters but not necessarily the same at distinct occurrences. The same remark holds for lower case letters.

\section{Preliminary results}

In this section, we give some fundamental facts about symmetric cones, Berezin transform, and related results.

\subsection{Symmetric cones, Bergman metric, and estimations of the determinant function}

It is well known that a symmetric cone $\Omega$ induces in $V \equiv \mathbb{R}^{n}$ a structure of Euclidean Jordan algebra, in which $\bar{\Omega}=\left\{x^{2}: x \in V\right\}$. We denote by $\mathbf{e}$ the identity element in $V$. Denote by $G(\Omega)$ the group of transformations of $\mathbb{R}^{n}$ leaving invariant the cone $\Omega$. It is also well known that the group $G(\Omega)$ acts transitively on $\Omega$. We denote by $H$ the subgroup of $G(\Omega)$ that acts simply transitively on $\Omega$, that is for $x, y \in \Omega$ there is a unique $h \in H$ such that $y=h x$. Observe that if we still denote by $\mathbb{R}^{n}$ the group of translations by vectors in $\mathbb{R}^{n}$, then the group $G(\mathcal{D})=\mathbb{R}^{n} \times H$ acts simply transitively on $\mathcal{D}$.

Recall that $\delta>0$,

$$
B_{\delta}(z)=\{w \in \mathcal{D}: d(z, w)<\delta\}
$$

is the Bergman ball centered at $z$ with radius $\delta$, where $d(\cdot, \cdot)$ is the Bergman distance (for a definition, see for example [13]).

We recall the following (see [2, Theorem 5.4]).

Lemma 2.1 Given $\delta \in(0,1)$, there exists a sequence $\left\{\zeta_{j}\right\}$ of points of $\mathcal{D}$ called $\delta$-lattice such that, if $B_{j}=B_{\delta}\left(\zeta_{j}\right)$ and $B_{j}^{\prime}=B_{\frac{\delta}{2}}\left(\zeta_{j}\right)$, then

(i) The balls $B_{j}^{\prime}$ are pairwise disjoint;

(ii) The balls $B_{j}$ cover $\mathcal{D}$ with finite overlapping, i.e., there is an integer $N$ (depending only on $\mathcal{D})$ such that each point of $\mathcal{D}$ belongs to at most $N$ of these balls.

We recall that

$$
\int_{B_{\delta}(\zeta)} \mathrm{d} V_{\nu}(z) \approx \int_{B_{\frac{\delta}{2}}(\zeta)} \mathrm{d} V_{\nu}(z) \approx C_{\delta} \Delta^{v+n / r}(\Im \zeta)
$$

for any $\zeta \in \mathcal{D}$.

We also recall that the measure $\mathrm{d} \lambda(z)=\Delta^{-2 n / r}(\Im z) \mathrm{d} V(z)$ is an invariant measure on $\mathcal{D}$ under the actions of $G(\mathcal{D})=\mathbb{R}^{n} \times H$.

Remark 2.2 Let $A>0$ be fixed. Assume that $0<\delta<A$. Then any $\delta$-lattice $\left\{\zeta_{j}\right\}$ admits a decomposition into a finite number of sequences $\left\{\zeta_{j_{k}}\right\}$ satisfying $d\left(\zeta_{j_{1}}, \zeta_{k_{2}}\right) \geq A$ for $j_{k_{1}} \neq j_{k_{2}}$ (see for example [19, Lemma 2.1]). 
We refer to [2, Theorem 5.6] for the following sampling theorem.

Lemma 2.3 Let $\left\{\zeta_{j}\right\}_{j \in \mathbb{N}}$ be a $\delta$-lattice in $\mathcal{D}, \delta \in(0,1)$. Then the following assertions hold.

(1) There is a positive constant $C_{\delta}$ such that every $f \in A_{v}^{p}(\mathcal{D})$ satisfies

$$
\left\|\left\{f\left(\zeta_{j}\right) \Delta^{\frac{1}{p}\left(v+\frac{n}{r}\right)}\left(\Im \zeta_{j}\right)\right\}\right\|_{l^{p}} \leq C_{\delta}|| f \|_{p, \nu} .
$$

(2) Conversely, if $\delta$ is small enough, there is a positive constant $C_{\delta}$ such that every $f \in A_{\nu}^{p}(\mathcal{D})$ satisfies

$$
\|f\|_{p, v} \leq C_{\delta}\left\|\left\{f\left(\zeta_{j}\right) \Delta^{\frac{1}{p}\left(v+\frac{n}{r}\right)}\left(\Im \zeta_{j}\right)\right\}\right\|_{l^{p}} .
$$

We have the following atomic decomposition with change of weight which is derived from [13, Theorem 3.2].

Theorem 2.4 Let $\sigma, v>\frac{n}{r}-1$. Assume that the operator $P_{\sigma}$ is bounded on $L_{v}^{2}(\mathcal{D})$ and let $\left\{\zeta_{j}\right\}_{j \in \mathbb{N}}$ be a $\delta$-lattice in $\mathcal{D}$. Then the following assertions hold.

(i) For every complex sequence $\left\{\lambda_{j}\right\}_{j \in \mathbb{N}}$ in $l^{2}$, the series

$$
\sum_{j} \lambda_{j} K_{\sigma}\left(z, \zeta_{j}\right) \Delta^{\sigma+\frac{1}{2}\left(\frac{n}{r}-v\right)}\left(\Im \zeta_{j}\right)
$$

is convergent in $A_{v}^{2}(\mathcal{D})$. Moreover, its sum $f$ satisfies the inequality

$\|f\|_{2, v} \leq C_{\delta}\left\|\left\{\lambda_{j}\right\}\right\|_{l^{2}}$

where $C_{\delta}$ is a positive constant.

(ii) For $\delta$ small enough, every function $f \in A_{v}^{2}(\mathcal{D})$ may be written as

$$
\begin{aligned}
& f(z)=\sum_{j} \lambda_{j} K_{\sigma}\left(z, \zeta_{j}\right) \Delta^{\sigma+\frac{1}{2}\left(\frac{n}{r}-v\right)}\left(\Im \zeta_{j}\right) \\
& \text { with } \\
& \left\|\left\{\lambda_{j}\right\}\right\|_{l^{2}} \leq C_{\delta}\|f\|_{2, v} \\
& \text { where } C_{\delta} \text { is a positive constant. }
\end{aligned}
$$

We remark that for $\sigma>\frac{n}{r}-1$ sufficiently large, $P_{\sigma}$ is bounded on $L_{v}^{2}(\mathcal{D})$ (see for example [17, Theorem 1.1]).

We will need the following consequence of the mean value theorem (see [2, Proposition 5.5]).

Lemma 2.5 There exists a constant $C>0$ such that for any $f \in \mathcal{H}(\mathcal{D})$ and $\delta \in(0,1]$, the following holds:

$$
|f(z)|^{p} \leq C \delta^{-n} \int_{B_{\delta}(z)}|f(\zeta)|^{p} \frac{\mathrm{d} V(\zeta)}{\Delta^{2 n / r}(\Im \zeta)} .
$$


We recall the following integrability conditions for the determinant function (see [2, Lemma 3.20]).

Lemma 2.6 Let $\alpha$ be real. Then the function $f(z)=\Delta^{-\alpha}\left(\frac{z+i t}{i}\right)$, with $t \in \Omega$, belongs to $L_{v}^{p}(\mathcal{D})$ if and only if $v>\frac{n}{r}-1$ and $p \alpha>v+2 \frac{n}{r}-1$. In this case,

$$
\|f\|_{p, v}^{p}=C_{\alpha, p} \Delta^{-p \alpha+\frac{n}{r}+v}(t) .
$$

The following integration by parts is a consequence of the Plancherel formula and Paley-Wiener characterization of $A_{v}^{2}(\mathcal{D})$ (see [2, Lemma 3.11]).

Lemma 2.7 Let $v>\frac{n}{r}-1$, Then for any $f, g \in A_{v}^{2}(\mathcal{D})$ and any integer $m \geq 0$, we have the formula

$$
\int_{\mathcal{D}} f(z) \overline{g(z)} \mathrm{d} V_{v}(z)=C_{v, m} \int_{\mathcal{D}} f(z) \overline{\square^{m} g(z)} \Delta^{m}(\Im z) \mathrm{d} V_{v}(z) .
$$

The following can also be proved using the Plancherel formula and Paley-Wiener characterization of $A_{v}^{2}(\mathcal{D})$.

Lemma 2.8 Let $v>\frac{n}{r}-1$, and let $m>0$ be an integer. Then there is a constant $C_{v, m}>0$ such that for any $f \in A_{v}^{2}(\mathcal{D})$,

$$
\|f\|_{2, v}=C_{v, m}\left\|\square^{m} f\right\|_{2,2 m+v} .
$$

We will be using the following Korányi's lemma.

Lemma 2.9 [6, Theorem 1.1] For every $\rho>0$, there is a constant $C_{\rho}>0$ such that

$$
\left|\frac{K(\zeta, z)}{K(\zeta, w)}-1\right| \leq C_{\rho} d(z, w)
$$

for all $\zeta, z, w \in \mathcal{D}$, with $d(z, w) \leq \rho$.

We close this subsection by recalling the following consequence of [2, Corollary 3.4] and the above Korányi's lemma for $\rho=1$.

Lemma 2.10 Let $v>\frac{n}{r}-1$. Then the following assertions hold.

(a) There is a positive constant $C_{v}$ such that for all $\delta \in(0,1], w \in \mathcal{D}$ and $z \in B_{\delta}(w)$, $V_{\nu}\left(B_{\delta}(w)\right)\left|k_{\nu}(z, w)\right|^{2} \leq C$.

(b) There is a positive constant $c$ such that if $\delta \in(0,1]$ is sufficiently small, then for any $w \in \mathcal{D}$ and for every $z \in B_{\delta}(w)$, we have

$V_{\nu}\left(B_{\delta}(w)\right)\left|k_{v}(z, w)\right|^{2} \geq c(1-C \delta)$.

In the latter, $C$ is the constant in Lemma 2.9 corresponding to $\rho=1$.

The following was obtained in [16, Lemma 3.1] for symmetric homogeneous domains in $\mathbb{C}^{n}$ and extended to the case of homogeneous Siegel domains of type II in [7, Lemma 5.1]. 
Lemma 2.11 Let $\beta>2 \frac{n}{r}-1$ and $\alpha>\beta+\frac{n}{r}-1$. Then for any $\varepsilon>0$, there exists $A_{\varepsilon}>0$ such that if $\left\{z_{j}=x_{j}+i y_{j}\right\}$ is a sequence of points of D satisfying $\inf _{j \neq k} d\left(z_{j}, z_{k}\right) \geq A_{\varepsilon}$, then for any integer $j$, the following estimate holds

$$
\sum_{\{k: k \neq j\}}\left|\Delta^{-\alpha}\left(z_{k}-\bar{z}_{j}\right)\right| \Delta^{\beta}\left(y_{k}\right) \leq \varepsilon \Delta^{-\alpha+\beta}\left(y_{j}\right) .
$$

Proof Let us give ourselves $A>1$. We assume that the sequence $\left\{z_{j}=x_{j}+i y_{j}\right\}$ is such that $d\left(z_{j}, z_{k}\right) \geq A$ for all $j \neq k$. We first observe with Lemma 2.5 that

$$
\left|\Delta^{-\alpha}\left(z_{k}-\bar{z}_{j}\right)\right| \leq C(1 / 3)^{-n} \int_{B_{k}^{\prime}}\left|\Delta^{-\alpha}\left(w-\bar{z}_{j}\right)\right| \frac{\mathrm{d} V(w)}{\Delta^{\frac{2 n}{r}}(\Im w)} .
$$

It follows that

$$
\begin{aligned}
S & :=\sum_{\{k: k \neq j\}}\left|\Delta^{-\alpha}\left(z_{k}-\bar{z}_{j}\right)\right| \Delta^{\beta}\left(y_{k}\right) \\
& \leq C(1 / 3)^{-n} \sum_{\{k: k \neq j\}} \int_{B_{k}^{\prime}}\left|\Delta^{-\alpha}\left(w-\bar{z}_{j}\right)\right| \Delta^{\beta}(\Im w) \frac{\mathrm{d} V(w)}{\Delta^{\frac{2 n}{r}(\mathfrak{w} w)}} \\
& =C(1 / 3)^{-n} \int_{\mathcal{D}}\left|\Delta^{-\alpha}\left(w-\bar{z}_{j}\right)\right| \Delta^{\beta}(\mathfrak{I} w) \frac{\mathrm{d} V(w)}{\Delta^{\frac{2 n}{r}}(\mathfrak{I} w)}
\end{aligned}
$$

where $\mathcal{B}:=\bigcup_{k \neq j} B_{k}^{\prime}$.

Now observe that if $w \in \mathcal{B}$, then $w \in B_{k}^{\prime}$ for some $k$ and so

$$
d\left(w, z_{k}\right)<\frac{A}{2}
$$

for $k \neq j$, and so

$$
d\left(w, z_{j}\right) \geq d\left(z_{j}, z_{k}\right)-d\left(w, z_{k}\right)>A-\frac{A}{2}=\frac{A}{2} .
$$

Let $g \in G(\mathcal{D})$ be the transformation such that $g(i \mathbf{e})=z_{j}$, and put $w=g(\zeta)$. Observe that for any $s \in \mathbb{R}$,

$$
\begin{aligned}
& \Delta^{s}(\mathfrak{I} w)=(\operatorname{Det} g)^{\frac{r}{n} s} \Delta^{s}(\mathfrak{I} \zeta)=\Delta^{s}\left(\mathfrak{I} z_{j}\right) \Delta^{s}(\Im \zeta), \\
& \Delta^{s}\left(w-\bar{z}_{j}\right)=\Delta^{s}(g(\zeta+i \mathbf{e}))=(\operatorname{Det} g)^{\frac{r}{n} s} \Delta^{s}(\zeta+i \mathbf{e})=\Delta^{s}\left(\mathfrak{I} z_{j}\right) \Delta^{s}(\zeta+i \mathbf{e})
\end{aligned}
$$

and

$$
\mathrm{d} V(w)=(\operatorname{Det} g)^{2} \mathrm{~d} V(\zeta)=\Delta^{2 \frac{n}{r}}\left(\Im z_{j}\right) \mathrm{d} V(\zeta)
$$

It follows that 


$$
\begin{aligned}
S & \leq C(1 / 3)^{-n} \int_{d\left(z_{j}, w\right)>A / 2}\left|\Delta^{-\alpha}\left(w-\bar{z}_{j}\right)\right| \Delta^{\beta}(\Im w) \frac{\mathrm{d} V(w)}{\Delta^{\frac{2 n}{r}}(\Im w)} \\
& \leq C(1 / 3)^{-n} \Delta^{-\alpha+\beta}\left(y_{j}\right) \int_{d(i e, \zeta)>A / 2}\left|\Delta^{-\alpha}(\zeta+i e)\right| \Delta^{\beta}(\Im \zeta) \frac{\mathrm{d} V(\zeta)}{\Delta^{\frac{2 n}{r}}(\Im \zeta)} .
\end{aligned}
$$

From the assumptions on $\alpha$ and $\beta$ together with Lemma 2.6, one has that the integral

$$
\int_{T_{\Omega}}\left|\Delta^{-\alpha}(\zeta+i e)\right| \Delta^{\beta}(\Im \zeta) \frac{\mathrm{d} V(\zeta)}{\Delta^{\frac{2 n}{r}}(\zeta)}
$$

converges. Hence, there exists $A_{\varepsilon}>0$ such that for all $A \geq A_{\varepsilon}$, the following inequality holds

$$
\int_{d(i e, \zeta)>A / 2}\left|\Delta^{-\alpha}(\zeta+i e)\right| \Delta^{\beta}(\Im \zeta) \frac{\mathrm{d} V(\zeta)}{\Delta^{\frac{2 n}{r}}(\Im \zeta)} \leq \frac{\varepsilon}{C} .
$$

The proof is complete.

\subsection{Averaging functions and Berezin transform}

The following was proved in [13] for $1 \leq p \leq \infty$. A careful observation of the proof of [13, Lemma 2.9] shows that the result extends to $0<p<1$.

Lemma 2.12 Let $0<p \leq \infty, v \in \mathbb{R}$, and $\delta, \beta \in(0,1)$. Assume that $\mu$ is a positive Borel measure on $\mathcal{D}$. Then the following assertions are equivalent.

(i) The function $\mathcal{D} \ni z \mapsto \frac{\mu\left(B_{\delta}(z)\right)}{\Delta^{v+\frac{n}{r}(\Im z)}}$ belongs to $L_{\nu}^{p}(\mathcal{D})$.

(ii) The function $\mathcal{D} \ni z \mapsto \frac{\mu\left(B_{\beta}(z)\right)}{\Delta^{v+\frac{n}{r}(\Im z)}}$ belongs to $L_{\nu}^{p}(\mathcal{D})$.

Note that, the above lemma allows flexibility on the choice of the radius of the ball. This fact is quite useful as seen in [13].

We have the following result.

Lemma 2.13 Let $0<p \leq 1, v>\frac{n}{r}-1, \beta, \delta \in(0,1)$. Let $\left\{\zeta_{j}\right\}_{j \in \mathbb{N}}$ be a $\delta$-lattice in $\mathcal{D}$, and let $\hat{\mu}_{\beta}$ and $\tilde{\mu}$ be in this order, the average function and the Berezin transform associated to the weight $v$. Then the following assertions are equivalent.

(i) $\hat{\mu}_{\beta} \in L^{p}(\mathcal{D}, \mathrm{d} \lambda)$.

(ii) $\left\{\hat{\mu}_{\delta}\left(\zeta_{j}\right)\right\}_{j \in \mathbb{N}} \in l^{p}$.

If moreover, $p>\frac{2 \frac{n}{r}-1}{v+\frac{n}{r}}$, then the above assertions are equivalent to

(iii) $\tilde{\mu} \in L^{p}(\mathcal{D}, \mathrm{d} \lambda)$. 
Proof The equivalence (i) $\Leftrightarrow$ (ii) follows as in [13, Lemma 2.12]. That (iii) $\Rightarrow$ (i) follows from the fact that for any $\delta \in(0,1)$, there exists a constant $C_{\delta}>0$ such that for any $z \in \mathcal{D}$,

$$
\hat{\mu}_{\beta}(z) \leq C_{\delta} \tilde{\mu}(z)
$$

(see [13, Lemma 2.8]). To finish the proof, let us prove that (ii) $\Rightarrow$ (iii). First using Lemma 2.9, we obtain

$$
\begin{aligned}
\tilde{\mu}(z) & :=\int_{\mathcal{D}}\left|K_{v}(z, w)\right|^{2} \Delta^{v+\frac{n}{r}}(\Im z) \mathrm{d} \mu(w) \\
& \leq \sum_{k} \int_{B_{k}}\left|K_{v}(z, w)\right|^{2} \Delta^{v+\frac{n}{r}}(\Im z) \mathrm{d} \mu(w) \\
& \leq C \sum_{k}\left|K_{v}\left(z, \zeta_{k}\right)\right|^{2} \Delta^{v+\frac{n}{r}}(\Im z) \mu\left(B_{k}\right) \\
& \leq C \sum_{k}\left|K_{v}\left(z, \zeta_{k}\right)\right|^{2} \Delta^{v+\frac{n}{r}}(\Im z) \Delta^{v+\frac{n}{r}}\left(\Im \zeta_{k}\right) \hat{\mu}_{\delta}\left(\zeta_{k}\right) .
\end{aligned}
$$

As $0<p \leq 1$, it follows that

$$
(\tilde{\mu}(z))^{p} \leq C \sum_{k}\left|K_{v}\left(z, \zeta_{k}\right)\right|^{2 p} \Delta^{p\left(v+\frac{n}{r}\right)}(\Im z) \Delta^{p\left(v+\frac{n}{r}\right)}\left(\Im \zeta_{k}\right)\left(\hat{\mu}_{\delta}\left(\zeta_{k}\right)\right)^{p} .
$$

Hence using that $p>\frac{2 \frac{n}{r}-1}{v+\frac{h}{r}}$ together with Lemma 2.6, we obtain

$$
\begin{aligned}
L & :=\int_{\mathcal{D}}(\tilde{\mu}(z))^{p} \mathrm{~d} \lambda(z) \\
& \leq C \sum_{k} \Delta^{p\left(v+\frac{n}{r}\right)}\left(\Im \zeta_{k}\right)\left(\hat{\mu}_{\delta}\left(\zeta_{k}\right)\right)^{p} \int_{\mathcal{D}}\left|K_{v}\left(z, \zeta_{k}\right)\right|^{2 p} \Delta^{p\left(v+\frac{n}{r}\right)-2 \frac{n}{r}}(\Im z) \mathrm{d} V(z) \\
& \leq C \sum_{k}\left(\hat{\mu}_{\delta}\left(\zeta_{k}\right)\right)^{p}<\infty .
\end{aligned}
$$

The proof is complete.

From the above proof, it is clear that the implication (iii) $\Rightarrow$ (i) is valid for every $p \in(0,1]$.

\subsection{Schatten class operators}

Let $\mathcal{H}_{1}$ and $\mathcal{H}_{2}$ be two Hilbert spaces. Let $\mathcal{B}\left(\mathcal{H}_{1}, \mathcal{H}_{2}\right)$ and $\mathcal{K}\left(\mathcal{H}_{1}, \mathcal{H}_{2}\right)$ denote the sets of bounded and compact operators from $\mathcal{H}_{1}$ to $\mathcal{H}_{2}$, respectively. It is well known that any operator $T \in \mathcal{K}\left(\mathcal{H}_{1}, \mathcal{H}_{2}\right)$ has a Schmidt decomposition, that is there exist orthonormal basis $\left\{e_{j}\right\}$ and $\left\{\sigma_{j}\right\}$ of $\mathcal{H}_{1}$ and $\mathcal{H}_{2}$ respectively and a sequence $\left\{\lambda_{j}\right\}$ of complex numbers converging to 0 such that

$$
T f=\sum_{j=0}^{\infty} \lambda_{j}\left\langle f, e_{j}\right\rangle \sigma_{j}, \quad f \in \mathcal{H}_{1} .
$$


For $0<p<\infty$, a compact operator $T$ with such a decomposition belongs to the Schatten-Von Neumann p-class $\mathcal{S}_{p}\left(\mathcal{H}_{1}, \mathcal{H}_{2}\right)$ if and only if

$$
\|T\|_{\mathcal{S}_{p}}=\left(\sum_{j=0}^{\infty}\left|\lambda_{j}\right|^{p}\right)^{\frac{1}{p}}<\infty
$$

When $\mathcal{H}_{1}=\mathcal{H}_{2}=\mathcal{H}$, we simply write $\mathcal{B}(\mathcal{H})=\mathcal{B}(\mathcal{H}, \mathcal{H})$ and $\mathcal{K}(\mathcal{H})=\mathcal{K}(\mathcal{H}, \mathcal{H})$ for the spaces of bounded and compact linear operators on $\mathcal{H}$ respectively. The norm on $\mathcal{H}$ will be denoted $\|\cdot\|_{\mathcal{H}}$ or simply $\|\cdot\|$ when there is no ambiguity. We recall that for any positive operator $T \in \mathcal{K}(\mathcal{H})$ there exist an orthonormal basis $\left\{e_{j}\right\}$ of $\mathcal{H}$ and a sequence of nonnegative numbers $\left\{\lambda_{j}\right\}$ that decreases to 0 such that

$$
T f=\sum_{j=0}^{\infty} \lambda_{j}\left\langle f, e_{j}\right\rangle e_{j}, \quad f \in \mathcal{H} .
$$

For $p=1, \mathcal{S}_{1}=\mathcal{S}_{1}(\mathcal{H})$ is the trace class and for $T \in \mathcal{S}_{1}$, the trace of $T$ is defined by

$$
\operatorname{Tr}(T)=\sum_{j=0}^{\infty}\left\langle T e_{j}, e_{j}\right\rangle
$$

where $\left\{e_{j}\right\}$ is any orthonormal basis of the Hilbert space $\mathcal{H}$.

It is known that a compact operator $\mathrm{T}$ on $\mathcal{H}$ belongs to the Schatten class $\mathcal{S}_{p}$ if and only if the positive operator $\left(T^{*} T\right)^{1 / 2}$ belongs to $\mathcal{S}_{p}$, where $T^{*}$ denotes the adjoint of $T$. In this case, we have $\|T\|_{\mathcal{S}_{p}}=\left\|\left(T^{*} T\right)^{1 / 2}\right\|_{\mathcal{S}_{p}}$. It is also well known that a positive $T$ belongs to $\mathcal{S}_{p}$ if and only if the operator $T^{p}$ belongs to the trace class $\mathcal{S}_{1}$. In this case, $\|T\|_{\mathcal{S}_{p}}=\left\|T^{p}\right\|_{\mathcal{S}_{1}}$.

We also recall that if $T$ is a compact operator on $\mathcal{H}$, and $p \geq 1$, then that $T \in \mathcal{S}_{p}$ is equivalent to

$$
\sum_{j}\left|\left\langle T e_{j}, e_{j}\right\rangle\right|^{p}<\infty
$$

for any orthonormal set $\left\{e_{j}\right\}$ in $\mathcal{H}$ (see [20, Theorem 1.27]). In particular, if $T \in \mathcal{S}_{1}$ is a positive operator, then $\|T\|_{\mathcal{S}_{1}}=\operatorname{Tr}(T)$.

The following can be found in [20, Corollary 1.32].

Lemma 2.14 Suppose that $T$ is a positive operator on $\mathcal{H}$, and that $\left\{e_{j}\right\}$ is an orthonormal basis on $\mathcal{H}$. Then if $0<p<1$ and

$$
\sum_{j=1}^{\infty}\left\langle T e_{j}, e_{j}\right\rangle^{p}<\infty
$$

then $T$ belongs to $\mathcal{S}_{p}$. 
We also observe the following (see [20, Proposition 1.30])

Lemma 2.15 Let $T$ be any bounded operator on $\mathcal{H}$ and assume that $A$ is bounded surjective operator on $\mathcal{H}$. Then $T$ belongs to $\mathcal{S}_{p}$ if and only if the operator $A^{*} T A$ belongs to $\mathcal{S}_{p}$.

Finally, we will need the following result (see [12, Lemma 5] and [20, Proposition 1.29])

Lemma 2.16 Let $T$ be any compact operator on $\mathcal{H}$ and let $\left\{e_{k}\right\}$ be an orthonormal basis of $\mathcal{H}$. Then for any $0<p \leq 2$, we have

$$
\|T\|_{\mathcal{S}_{p}}^{p} \leq \sum_{k} \sum_{j}\left|\left\langle T e_{k}, e_{j}\right\rangle\right|^{p} .
$$

\section{Schatten class membership of Toeplitz operators}

The aim of this section is to give criteria for Schatten class membership of Toeplitz operators on the weighted Bergman space $A_{v}^{2}(\mathcal{D})$.

\subsection{Proof of Theorem 1.2}

We start by proving the following which is actually the implication (ii) $\Rightarrow$ (i) in Theorem 1.2.

Lemma 3.1 Let $\mu$ be a positive Borel measure on $\mathcal{D}$, and $v>\frac{n}{r}-1$. Assume that $\frac{\frac{n}{r}-1}{v+\frac{n}{r}}<p<1$. Suppose that for any $\delta$-lattice $(\delta \in(0,1))\left\{\zeta_{j}\right\}_{j \in \mathbb{N}}$ in the Bergman metric of $\mathcal{D}$, the sequence $\left\{\hat{\mu}_{\delta}\left(\zeta_{j}\right)\right\}$ belongs to $l^{p}$, that is

$$
\sum_{j}\left(\frac{\mu\left(B_{j}\right)}{\Delta^{v+n / r}\left(\Im \zeta_{j}\right)}\right)^{p}<\infty .
$$

Then the Toeplitz operator $T_{\mu}$ belongs to the Schatten class $\mathcal{S}_{p}\left(A_{\nu}^{2}(\mathcal{D})\right.$ ). Moreover,

$$
\left\|T_{\mu}\right\|_{\mathcal{S}_{p}}^{p} \lesssim \sum_{j}\left(\frac{\mu\left(B_{j}\right)}{\Delta^{v+n / r}\left(\Im \zeta_{j}\right)}\right)^{p} .
$$

Proof Let $\sigma$ be large enough so that $P_{\sigma}$ is bounded on $L_{v}^{2}(\mathcal{D})$. Thanks to Lemma 2.12, we can suppose that $\delta$ is small enough so that any $f \in A_{v}^{2}(\mathcal{D})$ can represented as in Theorem 2.4. That is

$$
f(z)=\sum_{j} \lambda_{j} K_{\sigma}\left(z, \zeta_{j}\right) \Delta^{\sigma+\frac{1}{2}\left(\frac{n}{r}-v\right)}\left(\Im \zeta_{j}\right)
$$

with $\left\|\left\{\lambda_{j}\right\}\right\|_{l^{2}} \approx\|f\|_{2, v}$.

Let $\left\{e_{k}\right\}_{k \geq 1}$ be a fixed orthonormal basis on $A_{v}^{2}(\mathcal{D})$. Consider the operator $S: A_{v}^{2}(\mathcal{D}) \rightarrow A_{v}^{2}(\mathcal{D})$ defined by

$$
S\left(e_{k}\right)=f_{k}
$$


where

$$
f_{k}(z)=K_{\sigma}\left(z, \zeta_{k}\right) \Delta^{\sigma+\frac{1}{2}\left(\frac{n}{r}-v\right)}\left(\Im \zeta_{k}\right)
$$

Then it follows from Theorem 2.4 that $S$ is a bounded and surjective operator on $A_{v}^{2}(\mathcal{D})$. We know from Lemma 2.15 that the positive operator $T_{\mu}$ belongs to $\mathcal{S}_{p}\left(A_{v}^{2}(\mathcal{D})\right)$ if and only if $T=S^{*} T_{\mu} S$ belongs to $\mathcal{S}_{p}\left(A_{v}^{2}(\mathcal{D})\right.$ ). It follows from Lemma 2.14 that we only have to prove that

$$
L:=\sum_{k=1}^{\infty}\left\langle T e_{k}, e_{k}\right\rangle^{p}<\infty .
$$

We first observe that

$$
\left\langle T e_{k}, e_{k}\right\rangle=\left\langle T_{\mu} f_{k}, f_{k}\right\rangle=\int_{\mathcal{D}}\left|f_{k}(z)\right|^{2} \mathrm{~d} \mu(z) .
$$

Hence using Lemma 2.10, we obtain

$$
\begin{aligned}
\left\langle T e_{k}, e_{k}\right\rangle & \leq \sum_{j=1}^{\infty} \int_{B_{\delta}\left(\zeta_{j}\right)}\left|f_{k}(z)\right|^{2} \mathrm{~d} \mu(z) \\
& \leq C \sum_{j=1}^{\infty}\left|f_{k}\left(\zeta_{j}\right)\right|^{2} \mu\left(B_{\delta}\left(\zeta_{j}\right)\right) .
\end{aligned}
$$

Recalling that $0<p<1$, we then obtain

$$
\begin{aligned}
\left\langle T e_{k}, e_{k}\right\rangle^{p} & \leq C \sum_{j=1}^{\infty}\left|f_{k}\left(\zeta_{j}\right)\right|^{2 p}\left(\mu\left(B_{\delta}\left(\zeta_{j}\right)\right)\right)^{p} \\
& \approx \sum_{j=1}^{\infty}\left|f_{k}\left(\zeta_{j}\right)\right|^{2 p} \Delta^{p\left(v+\frac{n}{r}\right)}\left(\Im \zeta_{j}\right)\left(\hat{\mu}_{\delta}\left(\zeta_{j}\right)\right)^{p} .
\end{aligned}
$$

Thus

$$
\begin{aligned}
L & :=\sum_{k=1}^{\infty}\left\langle T e_{k}, e_{k}\right\rangle^{p} \\
& \leq C \sum_{k=1}^{\infty} \sum_{j=1}^{\infty}\left|f_{k}\left(\zeta_{j}\right)\right|^{2 p} \Delta^{p\left(v+\frac{n}{r}\right)}\left(\Im \zeta_{j}\right)\left(\hat{\mu}_{\delta}\left(\zeta_{j}\right)\right)^{p} \\
& \leq C \sum_{j=1}^{\infty} \Delta^{p\left(v+\frac{n}{r}\right)}\left(\Im \zeta_{j}\right)\left(\hat{\mu}_{\delta}\left(\zeta_{j}\right)\right)^{p} \sum_{k=1}^{\infty}\left|f_{k}\left(\zeta_{j}\right)\right|^{2 p} .
\end{aligned}
$$

Using the fact that each point in $\mathcal{D}$ belongs to at most $N$ balls $B_{k}$ and the condition $\frac{\frac{n}{r}-1}{v+\frac{n}{r}}<p<1$, and assuming $\sigma$ large enough so that 


$$
2 p\left(\sigma+\frac{1}{2}\left(\frac{n}{r}-v\right)\right)>2 \frac{n}{r}-1
$$

we obtain using Lemmas 2.5 and 2.6, the following for the inner sum

$$
\begin{aligned}
L_{j} & :=\sum_{k=1}^{\infty}\left|f_{k}\left(\zeta_{j}\right)\right|^{2 p} \\
& =\sum_{k=1}^{\infty}\left|K_{\sigma}\left(\zeta_{j}, \zeta_{k}\right)\right|^{2 p} \Delta^{2 p\left(\sigma+\frac{n}{r}-\frac{1}{2}\left(v+\frac{n}{r}\right)\right)}\left(\Im \zeta_{k}\right) \\
& \leq C \sum_{k=1}^{\infty} \int_{B_{\delta}\left(\zeta_{k}\right)}\left|K_{\sigma}\left(\zeta_{j}, z\right)\right|^{2 p} \Delta^{2 p\left(\sigma+\frac{1}{2}\left(\frac{n}{r}-v\right)\right)-2 \frac{n}{r}}(\Im z) \mathrm{d} V(z) \\
& \leq C N \int_{\mathcal{D}}\left|K_{\sigma}\left(\zeta_{j}, z\right)\right|^{2 p} \Delta^{2 p\left(\sigma+\frac{1}{2}\left(\frac{n}{r}-v\right)\right)-2 \frac{n}{r}}(\Im z) \mathrm{d} V(z) \\
& \leq C N \Delta^{-p\left(v+\frac{n}{r}\right)}\left(\Im \zeta_{j}\right) .
\end{aligned}
$$

Using the latter, we conclude that

$$
\begin{aligned}
L & :=\sum_{k=1}^{\infty}\left\langle T e_{k}, e_{k}\right\rangle^{p} \\
& \leq C \sum_{j=1}^{\infty}\left(\hat{\mu}_{\delta}\left(\zeta_{j}\right)\right)^{p}<\infty .
\end{aligned}
$$

We next prove the reverse of the above result. This corresponds to the implication (i) $\Rightarrow$ (ii) in Theorem 1.2.

Lemma 3.2 Let $\mu$ be a positive measure on $\mathcal{D}$. Assume that $T_{\mu} \in \mathcal{S}_{p}\left(A_{v}^{2}(\mathcal{D})\right)$ for some $\frac{2\left(\frac{n}{r}-1\right)}{v+\frac{n}{r}}<p<1$. Let $\left\{\zeta_{j}\right\}_{j \in \mathbb{N}}$ be a $\delta$-lattice in $\mathcal{D}$. Then the sequence $\left\{\hat{\mu}_{\delta}\left(\zeta_{j}\right)\right\}$ belongs to $l^{p}$. Moreover,

$$
\sum_{j}\left(\hat{\mu}_{\delta}\left(\zeta_{j}\right)\right)^{p} \lesssim\left\|T_{\mu}\right\|_{\mathcal{S}_{p}}^{p}
$$

Proof We start by considering $\sigma$ large enough so that $P_{\sigma}$ is bounded on $L_{v}^{2}(\mathcal{D})$ and $\alpha=p\left(\sigma+\frac{n}{r}\right)$ and $\beta=p\left(\sigma+\frac{1}{2}\left(\frac{n}{r}-v\right)\right)$ satisfy the conditions in Lemma 2.11. Let $\varepsilon>0$ and let $A_{\varepsilon}$ be as in Lemma 2.11. Following Remark 2.2, we may assume that our sequence $\left\{\zeta_{j}\right\}$ is such that $d\left(\zeta_{j}, \zeta_{k}\right)>A_{\varepsilon}$ for $j \neq k$. We further assume that $A_{\varepsilon}$ is large enough so that the corresponding balls $B_{k}$ are disjoint. Consider the following measure:

$$
\mathrm{d} \omega(z)=\sum_{k} \chi_{B_{k}}(z) \mathrm{d} \mu(z)
$$

Then $0 \leq \omega \leq \mu, \omega=\mu$ on each ball $B_{k}$. We also have the inequality $\left\|T_{\omega}\right\|_{\mathcal{S}_{p}}^{p} \leq\left\|T_{\mu}\right\|_{\mathcal{S}_{p}}^{p}$. Now as in the proof of the previous result, we fix an orthonormal basis $\left\{e_{k}\right\}$ of $A_{v}^{2}(\mathcal{D})$ and consider the same operator $S$ defined on $A_{v}^{2}(\mathcal{D})$ by $S\left(e_{k}\right)=f_{k}$ with 


$$
f_{k}(z)=K_{\sigma}\left(z, \zeta_{k}\right) \Delta^{\sigma+\frac{1}{2}\left(\frac{n}{r}-v\right)}\left(\Im \zeta_{k}\right) .
$$

We recall with Theorem 2.4 that $S$ is bounded and surjective on $A_{v}^{2}(\mathcal{D})$. Put again $T=S^{*} T_{\omega} S$. Then as $T_{\omega} \in \mathcal{S}_{p}\left(A_{v}^{2}(\mathcal{D})\right), T$ also belongs to $\mathcal{S}_{p}\left(A_{v}^{2}(\mathcal{D})\right)$ and we have

$$
\|T\|_{\mathcal{S}_{p}} \leq\|S\|^{2}\left\|T_{\omega}\right\|_{\mathcal{S}_{p}} \leq C\left\|T_{\mu}\right\|_{\mathcal{S}_{p}} .
$$

The main idea of the proof is to show that the $l^{p}$-norm of the sequence $\left\{\hat{\mu}_{\delta}\left(\zeta_{j}\right)\right\}$ is up to a constant a lower bound for $\|T\|_{\mathcal{S}_{p}}$. For this we decompose $T$ as $T=D+R$, where $D$ is the positive diagonal operator on $A_{v}^{2}(\mathcal{D})$ given by

$$
D f:=\sum_{k}\left\langle T e_{k}, e_{k}\right\rangle\left\langle f, e_{k}\right\rangle e_{k}, f \in A_{v}^{2}(\mathcal{D})
$$

and $R=T-D$. We observe that

$$
\|T\|_{\mathcal{S}_{p}}^{p} \geq\|D\|_{\mathcal{S}_{p}}^{p}-\|R\|_{\mathcal{S}_{p}}^{p}
$$

Hence, if we can prove that

$$
\|D\|_{\mathcal{S}_{p}}^{p} \geq c_{1} \sum_{j}\left(\hat{\mu}_{\delta}\left(\zeta_{j}\right)\right)^{p}
$$

and

$$
\|R\|_{\mathcal{S}_{p}}^{p} \leq c_{2} \sum_{j}\left(\hat{\mu}_{\delta}\left(\zeta_{j}\right)\right)^{p}
$$

with $c_{2}$ as small as we want, then the proof will be completed.

We start by estimating the diagonal operator $D$. As $D$ is positive, we have

$$
\begin{aligned}
\|D\|_{\mathcal{S}_{p}}^{p} & =\sum_{k}\left\langle T e_{k}, e_{k}\right\rangle^{p}=\sum_{k}\left\langle T_{\omega} f_{k}, f_{k}\right\rangle^{p} \\
& =\sum_{k}\left(\int_{\mathcal{D}}\left|f_{k}(z)\right|^{2} \mathrm{~d} \omega(z)\right)^{p} \\
& \geq \sum_{k}\left(\int_{B_{k}}\left|f_{k}(z)\right|^{2} \mathrm{~d} \omega(z)\right)^{p} \\
& =\sum_{k}\left(\int_{B_{k}}\left|f_{k}(z)\right|^{2} \mathrm{~d} \mu(z)\right)^{p} \\
& \asymp \sum_{k}\left(\hat{\mu}_{\delta}\left(\zeta_{k}\right)\right)^{p} .
\end{aligned}
$$

That is

$$
\|D\|_{\mathcal{S}_{p}}^{p} \geq c_{1} \sum_{j}\left(\hat{\mu}_{\delta}\left(\zeta_{j}\right)\right)^{p} .
$$

We now turn to the estimation of $\|R\|_{\mathcal{S}_{p}}^{p}$. First, using Lemma 2.16, we obtain 


$$
\begin{aligned}
\|R\|_{\mathcal{S}_{p}}^{p} & \leq \sum_{k} \sum_{j}\left|\left\langle R e_{j}, e_{k}\right\rangle\right|^{p} \\
& =\sum_{\{j, k: j \neq k\}}\left|\left\langle T e_{j}, e_{k}\right\rangle\right|^{p} \\
& =\sum_{\{j, k: j \neq k\}}\left|\left\langle T_{\omega} f_{j}, f_{k}\right\rangle\right|^{p} \\
& =\sum_{\{j, k: j \neq k\}}\left|\int_{\mathcal{D}} f_{j}(z) \overline{f_{k}}(z) \mathrm{d} \omega(z)\right|^{p} \\
& \leq \sum_{\{j, k: j \neq k\}}\left(\int_{\mathcal{D}}\left|f_{j}(z)\right| f_{k}(z) \mid \mathrm{d} \omega(z)\right)^{p} .
\end{aligned}
$$

As the balls $B_{l}$ are disjoint, using Lemma 2.10, we obtain

$$
\begin{aligned}
\int_{\mathcal{D}}\left|f_{j}(z)\right|\left|f_{k}(z)\right| \mathrm{d} \omega(z) & =\sum_{l} \int_{B_{l}}\left|f_{j}(z)\right|\left|f_{k}(z)\right| \mathrm{d} \mu(z) \\
& \leq C \sum_{i}\left|f_{j}\left(\zeta_{l}\right)\right|\left|f_{k}\left(\zeta_{l}\right)\right| \mu\left(B_{l}\right) \\
& \asymp \sum_{l}\left|f_{j}\left(\zeta_{l}\right)\right|\left|f_{k}\left(\zeta_{l}\right)\right| \Delta^{\nu+\frac{n}{r}}\left(\Im \zeta_{l}\right) \hat{\mu}_{\delta}\left(\zeta_{l}\right) .
\end{aligned}
$$

As $0<p<1$, it follows that

$$
\|R\|_{\mathcal{S}_{p}}^{p} \leq C \sum_{l} \Delta^{p\left(v+\frac{n}{r}\right)}\left(\Im \zeta_{l}\right)\left(\hat{\mu}_{\delta}\left(\zeta_{l}\right)\right)^{p} L_{l}
$$

where

$$
\begin{aligned}
L_{l}:= & \sum_{\{j, k: j \neq k\}}\left|f_{j}\left(\zeta_{l}\right)\right|^{p}\left|f_{k}\left(\zeta_{l}\right)\right|^{p} \\
= & \sum_{\{j, k: j \neq k\}}\left|\Delta^{-p\left(\sigma+\frac{n}{r}\right)}\left(\frac{\zeta_{j}-\bar{\zeta}_{l}}{i}\right)\right| \Delta^{p\left(\sigma+\frac{1}{2}\left(\frac{n}{r}-v\right)\right)}\left(\Im_{\zeta}\right) \\
& \times\left|\Delta^{-p\left(\sigma+\frac{n}{r}\right)}\left(\frac{\zeta_{k}-\bar{\zeta}_{l}}{i}\right)\right| \Delta^{p\left(\sigma+\frac{1}{2}\left(\frac{n}{r}-v\right)\right)}\left(\Im \zeta_{k}\right) \\
= & 2 L_{l}^{1}+L_{l}^{2}
\end{aligned}
$$

with

$$
L_{l}^{1}:=\Delta^{-\frac{p}{2}\left(v+\frac{n}{r}\right)}\left(\Im \zeta_{l}\right) \sum_{\{j: j \neq l\}}\left|\Delta^{-p\left(\sigma+\frac{n}{r}\right)}\left(\frac{\zeta_{j}-\bar{\zeta}_{l}}{i}\right)\right| \Delta^{p\left(\sigma+\frac{1}{2}\left(\frac{n}{r}-v\right)\right)}\left(\Im \zeta_{j}\right)
$$

and

$$
\begin{aligned}
L_{l}^{2}:= & \sum_{\{j, k: j \neq l \text { and } k \neq l\}}\left|\Delta^{-p\left(\sigma+\frac{n}{r}\right)}\left(\frac{\zeta_{j}-\bar{\zeta}_{l}}{i}\right)\right| \Delta^{p\left(\sigma+\frac{1}{2}\left(\frac{n}{r}-\nu\right)\right)}\left(\Im \zeta_{j}\right) \\
& \times\left|\Delta^{-p\left(\sigma+\frac{n}{r}\right)}\left(\frac{\zeta_{k}-\bar{\zeta}_{l}}{i}\right)\right| \Delta^{p\left(\sigma+\frac{1}{2}\left(\frac{n}{r}-v\right)\right)}\left(\Im \zeta_{k}\right) .
\end{aligned}
$$


Using Lemma 2.11, we obtain

$$
L_{l}^{1} \leq \varepsilon \Delta^{-p\left(v+\frac{n}{r}\right)}\left(\Im \zeta_{l}\right)
$$

and

$$
L_{l}^{2} \leq \varepsilon^{2} \Delta^{-p\left(v+\frac{n}{r}\right)}\left(\Im \zeta_{l}\right)
$$

It follows that

$$
\|R\|_{\mathcal{S}_{p}}^{p} \leq c_{2}\left(2 \varepsilon+\varepsilon^{2}\right) \sum_{j}\left(\hat{\mu}_{\delta}\left(\zeta_{j}\right)\right)^{p} .
$$

Hence

$$
\|T\|_{\mathcal{S}_{p}}^{p} \geq\left[\frac{c_{1}}{2}-c_{2}\left(2 \varepsilon+\varepsilon^{2}\right)\right] \sum_{j}\left(\hat{\mu}_{\delta}\left(\zeta_{j}\right)\right)^{p}
$$

Taking $\varepsilon$ small enough so that $\frac{c_{1}}{2}-c_{2}\left(2 \varepsilon+\varepsilon^{2}\right)>0$, we conclude that

$$
\sum_{j}\left(\hat{\mu}_{\delta}\left(\zeta_{j}\right)\right)^{p}<\infty
$$

The proof is complete.

We can now prove Theorem 1.2.

Proof of Theorem 1.2 We start by proving the necessity of the condition $p>\frac{2 \frac{n}{r}-1}{v+\frac{n}{r}}$ in assertion (iv). We recall that $\mathbf{e}$ is the identity element of $V$. We may suppose that $\mu\left(B_{1}(i \mathbf{e})\right)>0$ (if not change the radius of the Bergman ball). Then using Lemma 2.9, we obtain

$$
\begin{aligned}
\tilde{\mu}(z) & =\int_{\mathcal{D}}\left|k_{z}^{v}(w)\right|^{2} \mathrm{~d} \mu(w) \\
& \geq \int_{B_{1}(i \mathbf{e})}\left|k_{z}^{v}(w)\right|^{2} \mathrm{~d} \mu(w) \\
& \geq C \mu\left(B_{1}(i \mathbf{e})\right)\left|\Delta^{-\left(v+\frac{n}{r}\right)}\left(\frac{z}{i}+\mathbf{e}\right)\right|^{2} \Delta^{v+\frac{n}{r}}(\Im z) .
\end{aligned}
$$

It follows that if $\tilde{\mu}(z) \in L^{p}(\mathcal{D}, \mathrm{d} \lambda)$, then we should have

$$
\int_{\mathcal{D}}\left|\Delta^{-\left(v+\frac{n}{r}\right)}\left(\frac{z}{i}+\mathbf{e}\right)\right|^{2 p} \Delta^{p\left(v+\frac{n}{r}\right)}(\Im z) \frac{\mathrm{d} V(z)}{\Delta^{2 \frac{n}{r}}(\Im z)}<\infty
$$

which by Lemma 2.6 is possible only if $p\left(v+\frac{n}{r}\right)>2 \frac{n}{r}-1$.

Now the equivalences (ii) $\Leftrightarrow$ (iii) $\Leftrightarrow$ (iv) are from Lemma 2.13. The equivalence (i) $\Leftrightarrow$ (ii) is derived from Lemmas 3.1 and 3.2. The proof is complete. 


\subsection{Schatten class for general operators}

We consider here Schatten class criteria for an arbitrary operator defined on $A_{v}^{2}(\mathcal{D})$ with values in a Hilbert space $\mathcal{H}$. We denote by $\mathcal{B}\left(A_{v}^{2}(\mathcal{D}), \mathcal{H}\right)$ the set of bounded operators from $A_{v}^{2}(\mathcal{D})$ to $\mathcal{H}$. To avoid any confusion, we denote by $\langle\cdot, \cdot\rangle_{\mathcal{H}}$ and $\langle\cdot, \cdot\rangle_{v}$ the inner products in $\mathcal{H}$ and $A_{v}^{2}(\mathcal{D})$ respectively. We start with the Hilbert-Schmidt class $\mathcal{S}_{2}:=\mathcal{S}_{2}\left(A_{v}^{2}(\mathcal{D}), \mathcal{H}\right)$

Proposition 3.3 Let $T \in \mathcal{B}\left(A_{v}^{2}(\mathcal{D}), \mathcal{H}\right)$ then

$$
\|T\|_{\mathcal{S}_{2}\left(A_{v}^{2}(\mathcal{D}), \mathcal{H}\right)}^{2}=C_{n, m} \int_{\mathcal{D}}\left\|T\left(k_{z}^{v, m}\right)\right\|_{\mathcal{H}}^{2} \mathrm{~d} \lambda(z)
$$

for every integer $m \geq 0$.

Proof This result was proved in [18]. As the definition of Bergman spaces here is quite different, let us give a proof here for completeness. Let $\left\{e_{j}\right\}$ be an orthonormal basis of $\mathcal{H}$. Then using Lemma 2.8 , we obtain

$$
\begin{aligned}
\mathcal{I} & :=\int_{\mathcal{D}}\left\|T\left(K_{z}^{v, m}\right)\right\|_{\mathcal{H}}^{2} \Delta^{2 m+v-\frac{n}{r}}(\Im z) \mathrm{d} V(z) \\
& =\int_{\mathcal{D}} \sum_{j=0}^{\infty}\left|\left\langle T K_{z}^{v, m}, e_{j}\right\rangle_{\mathcal{H}}\right|^{2} \Delta^{2 m+v-\frac{n}{r}}(\Im z) \mathrm{d} V(z) \\
& =\sum_{j=0}^{\infty} \int_{\mathcal{D}}\left|\left\langle K_{z}^{v, m}, T^{*} e_{j}\right\rangle_{\nu}\right|^{2} \Delta^{2 m+v-\frac{n}{r}}(\Im z) \mathrm{d} V(z) \\
& =\sum_{j=0}^{\infty} \int_{\mathcal{D}}\left|\square_{z}^{m} T^{*} e_{j}(z)\right|^{2} \Delta^{2 m+v-\frac{n}{r}}(\Im z) \mathrm{d} V(z) \\
& =C_{v, m} \sum_{j=0}^{\infty} \int_{\mathcal{D}}\left|T^{*} e_{j}(z)\right|^{2} \mathrm{~d} V_{v}(z) \\
& =C_{v, m} \sum_{j=0}^{\infty}\left\|T^{*} e_{j}\right\|_{A_{v}^{2}}^{2} \\
& =C_{v, m}\left\|T^{*}\right\|_{\mathcal{S}_{2}}^{2}=C_{n, m}\|T\|_{\mathcal{S}_{2}}^{2} .
\end{aligned}
$$

We will deduce some results from the above one. The first one is the following which is obtained as in [18, Lemma 3.2].

Proposition 3.4 Suppose that $T \in \mathcal{B}\left(A_{v}^{2}(\mathcal{D}), \mathcal{H}\right)$. Let $m \geq 0$ be an integer. Then

(i) if $T \in \mathcal{S}_{p}\left(A_{v}^{2}(\mathcal{D}), \mathcal{H}\right)$ for $2<p<\infty$, then

$$
\int_{\mathcal{D}}\left\|T\left(k_{z}^{v, m}\right)\right\|_{\mathcal{H}}^{p} \mathrm{~d} \lambda(z) \leq C_{n, m}\|T\|_{\mathcal{S}_{p}\left(A_{\nu}^{2}(\mathcal{D}), \mathcal{H}\right)}^{p} .
$$

(ii) Iffor $0<p<2$,

$$
\int_{\mathcal{D}}\left\|T\left(k_{z}^{\nu, m}\right)\right\|_{\mathcal{H}}^{p} \mathrm{~d} \lambda(z)<\infty,
$$




$$
\begin{aligned}
& \text { then } T \in \mathcal{S}_{p}\left(A_{v}^{2}(\mathcal{D}), \mathcal{H}\right) \text {. Moreover, } \\
& \|T\|_{\mathcal{S}_{p}\left(A_{v}^{2}(\mathcal{D}), \mathcal{H}\right)}^{p} \leq C_{n, m} \int_{\mathcal{D}}\left\|T\left(k_{z}^{\nu, m}\right)\right\|_{\mathcal{H}}^{p} \mathrm{~d} \lambda(z) .
\end{aligned}
$$

We next have the following which is in fact implicit in the proof of the above result in [18].

Proposition 3.5 Suppose that $T \in \mathcal{B}\left(A_{v}^{2}(\mathcal{D})\right)$ is a positive operator. Let $m \geq 0$ be an integer. Then,

(i) if $T \in \mathcal{S}_{p}\left(A_{v}^{2}(\mathcal{D})\right)$ for $1 \leq p<\infty$, then

$$
\int_{\mathcal{D}}\left|\left\langle T\left(k_{z}^{v, m}\right), k_{z}^{v, m}\right\rangle_{\nu}\right|^{p} \mathrm{~d} \lambda(z) \leq C_{n, m}\|T\|_{\mathcal{S}_{p}\left(A_{\nu}^{2}(\mathcal{D})\right)}^{p} .
$$

(ii) Iffor $0<p \leq 1$,

$$
\begin{aligned}
& \int_{\mathcal{D}}\left|\left\langle T\left(k_{z}^{v, m}\right), k_{z}^{v, m}\right\rangle_{\nu}\right|^{p} \mathrm{~d} \lambda(z)<\infty, \\
& \text { then } T \in \mathcal{S}_{p}\left(A_{\nu}^{2}(\mathcal{D})\right) \text {. Moreover, } \\
& \|T\|_{\mathcal{S}_{p}\left(A_{\nu}^{2}(\mathcal{D})\right)}^{p} \leq C_{n, m} \int_{\mathcal{D}}\left|\left\langle T\left(k_{z}^{v, m}\right), k_{z}^{v, m}\right\rangle_{\nu}\right|^{p} \mathrm{~d} \lambda(z) .
\end{aligned}
$$

Proof From Proposition 3.3, we have that if $T \in \mathcal{S}_{1}\left(A_{v}^{2}(\mathcal{D})\right)$ is a positive operator, then

$$
\operatorname{Tr}(T)=\left\|T^{1 / 2}\right\|_{\mathcal{S}_{2}}^{2}=C_{n, m} \int_{\mathcal{D}}\left|\left\langle T\left(k_{z}^{\nu, m}\right), k_{z}^{\nu, m}\right\rangle_{\nu}\right| \mathrm{d} \lambda(z)
$$

Recalling that $\|T\|_{\mathcal{S}_{p}}^{p}=\operatorname{Tr}\left(T^{p}\right)$, the proof follows from the fact that for any unit vector(see [20]) $g \in L^{2}(\mathcal{D})$, we have

$$
\langle T g, g\rangle_{\nu}^{p} \leq\left\langle T^{p} g, g\right\rangle_{\nu}, \quad \text { if } p \geq 1
$$

and

$$
\left\langle T^{p} g, g\right\rangle_{v} \leq\langle T g, g\rangle_{v}^{p} \quad \text { if } \quad 0<p \leq 1 .
$$

\subsection{Proof of Theorem 1.3}

In this subsection, we establish a reproducing kernel thesis for $T_{\mu}$. First taking $T=T_{\mu}$ in Proposition 3.5, we obtain the following corollary.

Corollary 3.6 Let $\mu$ be a positive measure on $\mathcal{D}$, and let $m \geq 0$ be an integer. Then the following assertions hold.

(i) If $_{\mu} \in \mathcal{S}_{p}\left(A_{\nu}^{2}(\mathcal{D})\right)$ for $1 \leq p<\infty$, then

$$
\int_{\mathcal{D}}\left\langle T_{\mu}\left(k_{z}^{\nu, m}\right), k_{z}^{\nu, m}\right\rangle_{\nu}^{p} \mathrm{~d} \lambda(z) \leq C_{n, m}\left\|T_{\mu}\right\|_{\mathcal{S}_{p}\left(A_{\nu}^{2}(\mathcal{D})\right)}^{p} .
$$


(ii) If for $0<p \leq 1$,

$$
\begin{aligned}
& \int_{\mathcal{D}}\left\langle T_{\mu}\left(k_{z}^{v, m}\right), k_{z}^{v, m}\right\rangle_{\nu}^{p} \mathrm{~d} \lambda(z)<\infty, \\
& \text { then } T_{\mu} \in \mathcal{S}_{p}\left(A_{v}^{2}(\mathcal{D})\right) . \text { Moreover, } \\
& \left\|T_{\mu}\right\|_{\mathcal{S}_{p}\left(A_{v}^{2}(\mathcal{D})\right)}^{p} \leq C_{n, m} \int_{\mathcal{D}}\left\langle T_{\mu}\left(k_{z}^{v, m}\right), k_{z}^{v, m}\right\rangle_{\nu}^{p} \mathrm{~d} \lambda(z) .
\end{aligned}
$$

We next prove the following sufficient condition.

Lemma 3.7 Let $1 \leq p<\infty$, and let $m \geq 0$ be an integer. Assume that $\mu$ is a positive measure on $\mathcal{D}$. Then if the Toeplitz operator $T_{\mu}$ satisfies

$$
\int_{\mathcal{D}}\left\langle T_{\mu}\left(k_{z}^{v, m}\right), k_{z}^{v, m}\right\rangle_{\nu}^{p} \mathrm{~d} \lambda(z)<\infty
$$

then $T_{\mu} \in \mathcal{S}_{p}\left(A_{v}^{2}(\mathcal{D})\right)$. Moreover,

$$
\left\|T_{\mu}\right\|_{\mathcal{S}_{p}\left(A_{\nu}^{2}(\mathcal{D})\right)}^{p} \leq C_{n, m} \int_{\mathcal{D}}\left\langle T_{\mu}\left(k_{z}^{v, m}\right), k_{z}^{v, m}\right\rangle_{\nu}^{p} \mathrm{~d} \lambda(z)
$$

Proof By [20, Theorem 1.27], as $p \geq 1$, we only need to prove that there is positive constant $C$ such that for any orthonormal sequence $\left\{e_{k}\right\}$ on $A_{v}^{2}(\mathcal{D})$,

$$
\sum_{k}\left\langle T_{\mu} e_{k}, e_{k}\right\rangle_{\nu}^{p} \leq C \int_{\mathcal{D}}\left\langle T_{\mu}\left(k_{z}^{v, m}\right), k_{z}^{\nu, m}\right\rangle_{\nu}^{p} \mathrm{~d} \lambda(z)=C \int_{\mathcal{D}}\left(\tilde{\mu}^{m}(z)\right)^{p} \mathrm{~d} \lambda(z) .
$$

We recall our notation

$$
\tilde{\mu}^{m}(z):=\left\langle T_{\mu} k_{z}^{v, m}, k_{z}^{v, m}\right\rangle_{\nu}=\int_{\mathcal{D}}\left|k_{z}^{v, m}(w)\right|^{2} \mathrm{~d} \mu(w) .
$$

We start by noting that by Lemma 2.5 , we have

$$
\left|e_{k}(z)\right|^{2} \leq C \delta^{-n} \int_{B_{\delta}(z)}\left|e_{k}(w)\right|^{2} \mathrm{~d} \lambda(w) .
$$

It follows from this and Lemma 2.10 that

$$
\left|e_{k}(z)\right|^{2} \leq C \int_{\mathcal{D}}\left|e_{k}(w)\right|^{2}\left|k_{z}^{v, m}(w)\right|^{2} \mathrm{~d} V_{v}(w)
$$

Hence

$$
\begin{aligned}
\left\langle T_{\mu} e_{k}, e_{k}\right\rangle_{\nu} & =\int_{\mathcal{D}}\left|e_{k}(z)\right|^{2} \mathrm{~d} \mu(z) \\
& \leq \int_{\mathcal{D}}\left|e_{k}(w)\right|^{2} \tilde{\mu}^{m}(w) \mathrm{d} V_{\nu}(w)
\end{aligned}
$$


and so using Hölder's inequality, that the $e_{k}$ are orthonormal and

$$
\sum_{k}\left|e_{k}(z)\right|^{2}=K_{v}(z, z)
$$

we obtain

$$
\begin{aligned}
\sum_{k}\left\langle T_{\mu} e_{k}, e_{k}\right\rangle_{v}^{p} & \leq \sum_{k}\left(\int_{\mathcal{D}}\left|e_{k}(w)\right|^{2} \tilde{\mu}^{m}(w) \mathrm{d} V_{v}(w)\right)^{p} \\
& \leq \int_{\mathcal{D}}\left(\tilde{\mu}^{m}(w)\right)^{p}\left(\sum_{k}\left|e_{k}(w)\right|^{2}\right) \mathrm{d} V_{v}(w) \\
& \leq \int_{\mathcal{D}}\left(\tilde{\mu}^{m}(w)\right)^{p} K_{v}(w, w) \mathrm{d} V_{v}(w) \\
& \lesssim \int_{\mathcal{D}}\left(\tilde{\mu}^{m}(w)\right)^{p} \mathrm{~d} \lambda(w) .
\end{aligned}
$$

The proof is complete.

We now prove the following necessary condition.

Lemma 3.8 Let $m \geq 0$ be an integer such that $\max \left\{\frac{2 \frac{n}{r}-1}{v+\frac{n}{r}+2 m}, \frac{\frac{n}{r}-1}{v+\frac{n}{r}}\right\}<p \leq 1$. Assume that $\mu$ is a positive measure on $\mathcal{D}$. Then if the Toeplitz operator $T_{\mu}$ belongs to the Schatten class $\mathcal{S}_{p}\left(A_{v}^{2}(\mathcal{D})\right.$, then

$$
\int_{\mathcal{D}}\left\langle T_{\mu}\left(k_{z}^{v, m}\right), k_{z}^{v, m}\right\rangle_{\nu}^{p} \mathrm{~d} \lambda(z)<\infty
$$

Moreover,

$$
\int_{\mathcal{D}}\left\langle T_{\mu}\left(k_{z}^{v, m}\right), k_{z}^{v, m}\right\rangle_{\nu}^{p} \mathrm{~d} \lambda(z) \lesssim\left\|T_{\mu}\right\|_{\mathcal{S}_{p}\left(A_{\nu}^{2}(\mathcal{D})\right)}^{p}
$$

Proof Assume that the Toeplitz operator $T_{\mu}$ belongs to the Schatten class $\mathcal{S}_{p}\left(A_{v}^{2}(\mathcal{D})\right.$. Then by Lemma 3.2, this implies that for any $\delta$-lattice $\left\{\zeta_{k}\right\}$ of points of $\mathcal{D}$, the sequence $\left\{\hat{\mu}_{\delta}\left(\zeta_{k}\right)\right\}$ belongs to $l^{p}$ with

$$
\sum_{k}\left(\hat{\mu}_{\delta}\left(\zeta_{k}\right)\right)^{p} \lesssim\left\|T_{\mu}\right\|_{\mathcal{S}_{p}}^{p}
$$

It follows that to prove the above lemma, it is enough to prove that there is positive constant $C$ such that for any $\delta$-lattice $\left\{\zeta_{k}\right\}$ of points of $\mathcal{D}$,

$$
L:=\int_{\mathcal{D}}\left\langle T_{\mu}\left(k_{z}^{v, m}\right), k_{z}^{v, m}\right\rangle_{\nu}^{p} \mathrm{~d} \lambda(z) \leq C \sum_{k}\left(\hat{\mu}_{\delta}\left(\zeta_{k}\right)\right)^{p} .
$$

Recalling that $0<p \leq 1$ and using Lemma 2.9, we first obtain 


$$
\begin{aligned}
L & =\int_{\mathcal{D}}\left(\int_{\mathcal{D}}\left|k_{z}^{v, m}(w)\right|^{2} \mathrm{~d} \mu(w)\right)^{p} \mathrm{~d} \lambda(z) \\
& \leq \int_{\mathcal{D}}\left(\sum_{k} \int_{B_{k}}\left|k_{z}^{\nu, m}(w)\right|^{2} \mathrm{~d} \mu(w)\right)^{p} \mathrm{~d} \lambda(z) \\
& \leq C \int_{\mathcal{D}}\left(\sum_{k}\left|k_{z}^{v, m}\left(\zeta_{k}\right)\right|^{2} \mu\left(B_{k}\right)\right)^{p} \mathrm{~d} \lambda(z) \\
& \leq C \int_{\mathcal{D}}\left(\sum_{k}\left|K_{z}^{v, m}\left(\zeta_{k}\right)\right|^{2} \mu\left(B_{k}\right)\right)^{p} \frac{\Delta^{p\left(v+2 m+\frac{n}{r}\right)}(\Im z)}{\Delta^{2 \frac{n}{r}}(\Im z)} \mathrm{d} V(z) \\
& \leq C \sum_{k}\left(\mu\left(B_{k}\right)\right)^{p} \int_{\mathcal{D}}\left|K_{z}^{v, m}\left(\zeta_{k}\right)\right|^{2 p} \frac{\Delta^{p\left(v+2 m+\frac{n}{r}\right)}(\Im z)}{\Delta^{2 \frac{n}{r}}(\Im z)} \mathrm{d} V(z) .
\end{aligned}
$$

The condition on $p$ and Lemma 2.6 give us

$$
\int_{\mathcal{D}}\left|K_{z}^{\nu, m}\left(\zeta_{k}\right)\right|^{2 p} \Delta^{p\left(v+2 m+\frac{n}{r}\right)-2 \frac{n}{r}} \mathrm{~d} V(z)=C \Delta^{-p\left(v+\frac{n}{r}\right)}\left(\Im \zeta_{k}\right) .
$$

We then conclude that

$$
\begin{aligned}
L & \leq C \sum_{k}\left(\mu\left(B_{k}\right)\right)^{p} \Delta^{-p\left(v+\frac{n}{r}\right)}\left(\Im \zeta_{k}\right) \\
& \approx \sum_{k}\left(\hat{\mu}_{\delta}\left(\zeta_{k}\right)\right)^{p} .
\end{aligned}
$$

The proof is complete.

Theorem 1.3 clearly follows from Corollary 3.6, Lemma 3.7 and Lemma 3.8.

\section{Application to Cesàro-type operators}

Let us consider

$$
\mathcal{N}_{n}:=\left\{F \in \mathcal{H}(\mathcal{D}): \square^{n} F=0\right\}
$$

and set

$$
\mathcal{H}_{n}(\mathcal{D})=\mathcal{H}(\mathcal{D}) / \mathcal{N}_{n}
$$

We define for $g \in \mathcal{H}(\mathcal{D})$ the operator $T_{g}: \mathcal{H}(\mathcal{D}) \rightarrow \mathcal{H}_{n}(\mathcal{D})$ as follows: for $f \in \mathcal{H}(\mathcal{D}), T_{g} f$ is the equivalence class of the solutions $F$ of the equation

$$
\square^{n} F=f \square^{n} g .
$$

The operator $T_{g}$ was called in [13] Cesàro-type operator.

We consider in this part, criteria for Schatten class membership of the Cesàro-type operator above on the weighted Bergman space $A_{v}^{2}(\mathcal{D})$. In fact a characterization of Schatten classes for this operator was obtained in [13] for the range $2 \leq p \leq \infty$. Our aim here is to extend this result to the range $1 \leq p<2$. We refer to $[8,12,21,22]$ for the corresponding results on some classical domains. 
Let us recall that the Besov space $\mathcal{B}^{p}(\mathcal{D})$ is the subspace of $\mathcal{H}_{n}(\mathcal{D})$ consisting of equivalence classes $f$ such that the function $\Delta^{n} \square^{n} f$ defined by $\left(\Delta^{n} \square^{n} f\right)(z):=\Delta^{n}(\mathfrak{I} z) \square^{n} f(z)$ belongs to $L^{p}(\mathcal{D}, \mathrm{d} \lambda)$. For $f \in \mathcal{B}^{p}(\mathcal{D})$, we write $\|f\|_{\mathcal{B}^{p}} ;=\left\|\Delta^{n} \square^{n} f\right\|_{L^{p}(\mathcal{D}, \mathrm{d} \lambda)}$. For more on Besov spaces of tube domains over symmetric cones, we refer the reader to [3, 4].

We now obtain the following.

Theorem 4.1 Let $1 \leq p<2, v>\frac{n}{r}-1$. If $g$ is a given holomorphic function in $\mathcal{D}$, then the Cesàro-type operator $T_{g}$ belongs to $\mathcal{S}_{p}\left(A_{v}^{2}(\mathcal{D})\right.$ ) if and only if $g \in \mathcal{B}^{p}(\mathcal{D})$. Moreover,

$$
\left\|T_{g}\right\|_{\mathcal{S}_{p}\left(A_{v}^{2}(\mathcal{D})\right)} \approx\|g\|_{\mathcal{B}^{p}} .
$$

Proof Let us first assume that $T_{g} \in \mathcal{S}_{p}\left(A_{v}^{2}(\mathcal{D})\right)$. Then by Proposition 3.5, we have that

$$
\int_{\mathcal{D}}\left|\left\langle T_{g}\left(k_{z}^{\nu, n}\right), k_{z}^{\nu, n}\right\rangle_{\nu}\right|^{p} \mathrm{~d} \lambda(z) \leq C\left\|T_{g}\right\|_{\mathcal{S}_{p}\left(A_{\nu}^{2}(\mathcal{D})\right)}^{p} .
$$

Using Lemma 2.7 and reproducing formula, we obtain

$$
\begin{aligned}
\left\|T_{g}\right\|_{\mathcal{S}_{p}\left(A_{\nu}^{2}(\mathcal{D})\right)}^{p} & \geq C \int_{\mathcal{D}}\left|\left\langle T_{g}\left(k_{z}^{v, n}\right), k_{z}^{v, n}\right\rangle_{\nu}\right|^{p} \mathrm{~d} \lambda(z) \\
& =C_{n} \int_{\mathcal{D}}\left|\left\langle\square^{n}\left(T_{g} k_{z}^{v, n}(\cdot)\right), k_{z}^{v, n}(\cdot)\right\rangle_{\nu+n}\right|^{p} \mathrm{~d} \lambda(z) \\
& =C_{n} \int_{\mathcal{D}}\left|\left\langle\square^{n} g(\cdot) k_{z}^{v, n}(\cdot), K_{z}^{v, n}(\cdot)\right\rangle_{\nu+n}\right|^{p} \Delta^{\frac{p}{2}\left(v+\frac{n}{r}+2 n\right)}(\Im z) \mathrm{d} \lambda(z) \\
& =C_{n} \int_{\mathcal{D}}\left|\left(\square^{n} g(z)\right) k_{z}^{v, n}(z)\right|^{p} \Delta^{\frac{p}{2}\left(v+\frac{n}{r}+2 n\right)}(\Im z) \mathrm{d} \lambda(z) \\
& =C_{n} \int_{\mathcal{D}}\left|\Delta^{n}(\Im z) \square^{n} g(z)\right|^{p} \mathrm{~d} \lambda(z) .
\end{aligned}
$$

Hence $g \in \mathcal{B}^{p}(\mathcal{D})$ if $T_{g} \in \mathcal{S}_{p}\left(A_{v}^{2}(\mathcal{D})\right.$ ).

Now assume that $g \in \mathcal{B}^{p}(\mathcal{D})$. We consider the following measure

$$
\mathrm{d} \mu(z)=\left|\square^{n} g(z)\right|^{2} \Delta^{2 n+v-n / r}(\Im z) \mathrm{d} V(z) .
$$

We first observe the following. Let $\delta \in(0,1]$ be small enough and let $\left\{\zeta_{k}\right\}$ be a $\delta$-lattice of points of $\mathcal{D}$. Using Lemmas 2.3, 2.5, and 2.9, we obtain

$$
\begin{aligned}
\int_{\mathcal{D}}\left|\Delta^{n}(\Im z) \square^{n} g(z)\right|^{p} \mathrm{~d} \lambda(z) & \approx \sum_{j}\left(\left|\square^{n} g\left(\zeta_{j}\right)\right|^{2} \Delta^{2 n}\left(\Im \zeta_{j}\right)\right)^{p / 2} \\
& \lesssim \sum_{j}\left(\int_{B_{j}}\left|\square^{n} g(z)\right|^{2} \Delta^{2 n}(\Im z) \frac{\mathrm{d} V(z)}{\Delta^{2 n / r}(\Im z)}\right)^{p / 2} \\
& \approx \sum_{j}\left(\frac{1}{\Delta^{v+n / r}\left(\Im \zeta_{j}\right)} \int_{B_{j}} \mathrm{~d} \mu(z)\right)^{p / 2} \\
& =\sum_{j}\left(\frac{\mu\left(B_{j}\right)}{\Delta^{v+n / r}\left(\Im \zeta_{j}\right)}\right)^{p / 2}
\end{aligned}
$$


That is

$$
\int_{\mathcal{D}}\left|\Delta^{n}(\Im z) \square^{n} g(z)\right|^{p} \mathrm{~d} \lambda(z) \approx \sum_{j}\left(\frac{\mu\left(B_{j}\right)}{\Delta^{v+n / r}\left(\Im \zeta_{j}\right)}\right)^{p / 2}
$$

We next observe the following. Let $\left\{e_{j}\right\}$ be any orthonormal basis of $A_{v}^{2}(\mathcal{D})$. Then using Lemma 2.7, the definition of $T_{g}$ and Hölder's inequality, we obtain

$$
\begin{aligned}
\left\langle T_{g} e_{j}, e_{j}\right\rangle_{v}^{2} & =C_{n, v}\left\langle\square^{n} T_{g} e_{j}, e_{j}\right\rangle_{v+n}^{2} \\
& =C_{n, v}\left\langle\Delta^{n} \square^{n} T_{g} e_{j}, e_{j}\right\rangle_{v}^{2} \\
& \leq C_{n, v} \int_{\mathcal{D}}\left|e_{j}(z)\right|^{2} \mathrm{~d} \mu(z) \\
& =C_{n, v}\left\langle T_{\mu} e_{j}, e_{j}\right\rangle_{v} .
\end{aligned}
$$

It follows that

$$
\sum_{j}\left|\left\langle T_{g} e_{j}, e_{j}\right\rangle_{v}\right|^{p} \leq C_{n, v} \sum_{j}\left\langle T_{\mu} e_{j}, e_{j}\right\rangle_{v}^{p / 2}
$$

Hence, by (11), as $p \geq 1$, to prove that $T_{g}$ belongs to $\mathcal{S}_{p}\left(A_{v}^{2}(\mathcal{D})\right)$, it suffices by (13) to prove that

$$
\sum_{j}\left\langle T_{\mu} e_{j}, e_{j}\right\rangle_{\nu}^{p / 2}<\infty
$$

This follows from Lemma 3.1, using that as $g \in \mathcal{B}^{p}(\mathcal{D})$, we have by (12) that

$$
\sum_{j}\left(\frac{\mu\left(B_{j}\right)}{\Delta^{v+n / r}\left(\Im \zeta_{j}\right)}\right)^{p / 2}<\infty
$$

The proof is complete.

\section{Conclusion and further observations}

In this paper, we obtained some characterizations of Schatten class $\mathcal{S}_{p}\left(A_{v}^{2}(\mathcal{D})\right.$ ) of Toeplitz operators on the Bergman space $A_{v}^{2}(\mathcal{D})$ where $\mathcal{D}$ is a tube domain over a symmetric cone, for $0<p \leq 1$. We observe that in our first main result Theorem 1.2, depending on the assertion, there are some restrictions on the exponent $p$ of the Schatten class that are due to our method of proof that involves integrability conditions of the Bergman kernel. As a consequence, our result does not cover the whole range $0<p \leq 1$.

We proved that the condition $p>\frac{2 \frac{n}{r}-1}{v+\frac{n}{r}}$ in assertion (iv) of Theorem 1.2 is sharp, meaning that one can not go below the critical index $\frac{2 \frac{n}{r}-1}{v+\frac{n}{r}}$. Unfortunately, we do not know if the restrictions in the other assertions in the same theorem are sharp; for example, does assertion (ii) still hold for $\frac{\frac{n}{r}-1}{v+\frac{n}{r}} \leq p \leq \frac{2\left(\frac{n}{r}-1\right)}{v+\frac{n}{r}}$ ?

Another interesting question is to know if there are other characterizations of Schatten class of Toeplitz operators considered in this paper other than those given in Theorems 1.2 and 1.3 .

We note that although all the restrictions on our results, they allow us to complete Schatten class characterization of some Cesàro-type operators introduced in [13]. 
The author would like to thank the reviewer for carefully reading the manuscript and making several observations that improved the presentation of this paper.

\section{Authors' contributions}

BFS's contribution from this work is comprised in the characterization of Schatten class Toeplitz operators on weighted Bergman spaces of tube domains over symmetric for small exponents. This work is the first to the best of my knowledge as borne out in the originality in the proof provided, hitherto unknown, which heavily simplifies the known proofs for the unit ball. The author read and approved the final manuscript.

\section{Competing interests}

The author declares that he has no competing interests regarding this work.

\section{Publisher's Note}

Springer Nature remains neutral with regard to jurisdictional claims in published maps and institutional affiliations.

Received: 19 October 2017 Accepted: 30 July 2018

Published online: 20 August 2018

\section{References}

1. Arroussi, H., Park, I., Pau, J.: Schatten class Toeplitz operators acting on large weighted Bergman spaces. Studia Math. 229(3), 203-221 (2015)

2. Békollé, D., Bonami, A., Garrigós, G., Nana, C., Peloso, M., Ricci, F.: Lecture notes on Bergman projectors in tube domains over cones: an analytic and geometric viewpoint. IMHOTEP 5 (2004), Exposé I, Proceedings of the International Workshop in Classical Analysis, Yaoundé 2001

3. Békollé, D., Bonami, A., Garrigós, G., Ricci, F.: Littlewood-Paley decompositions related to symmetric cones and Bergman projections in tube domains. Proc. London Math. Soc. 89, 317-360 (2004)

4. Békollé, D., Bonami, A., Garrigós, G., Ricci, F., Sehba, B.: Hardy-type inequalities and analytic Besov spaces in tube domains over symmetric cones. J. Reine Angew. Math. 647, 25-56 (2010)

5. Békollé, D., Bonami, A., Peloso, M., Ricci, F.: Boundedness of weighted Bergman projections on tube domains over light cones. Math. Z. 237, 31-59 (2001)

6. Békollé, D., Ishi, H., Nana, C.: Korányi's lemma for homogeneous Siegel domains of type II. Applications and extended results. Bull. Aust. Math. Soc. 90, 77-89 (2014)

7. Békollé, D., Temgoua, A.: Molecular decompositions and interpolation. Integ. Equat. Oper. Theor. 31, 150-177 (1998)

8. Constantin, O.: Carleson embeddings and some classes of operators on weighted Bergman spaces. J. Math. Anal. Appl. 365, 668-682 (2010)

9. Debertol, D.: Besov spaces and boundedness of weighted Bergman projections over symmetric tube domains. Università di Genova, Politecnico di Torino (April, Dottorato di Ricerca in Matematica (2003)

10. Faraut, J., Korányi, A.: Analysis on symmetric cones. Clarendon Press, Oxford (1994)

11. Isralowitz, J., Virtanen, J., Wolf, L.: Schatten class Toeplitz operators on generalized Fock spaces. J. Math. Anal. Appl. 421(1), 329-337 (2015)

12. Luecking, D.: Trace ideal criteria for Toeplitz operators. J. Funct. Anal. 73(2), 345-368 (1987)

13. Nana, C., Sehba, B.F.: Carleson embeddings and two operators on Bergman spaces of tube domains over symmetric cones. Integr. Equ. Oper. Theory 83(2), 151-178 (2015)

14. Olivier, R., Pascuas, D.: Toeplitz operators on doubling Fock spaces (English summary). J. Math. Anal. Appl. 435(2), 1426-1457 (2016)

15. Pau, J.: A remark on Schatten class of Toeplitz operators on Bergman spaces. Proc. Am. Math. Soc. 142(8), 2763-2768 (2014)

16. Rochberg, R.: Interpolation by functions in Bergman spaces. Michigan Math. J. 83, 229-236 (1982)

17. Sehba, B.F.: Bergman type operators in tubular domains over symmetric cones. Proc. Edin. Math. Soc. 52(2), 529-544 (2009)

18. Sehba, B.F.: Hankel operators on Bergman spaces of tube domains over symmetric cones. Integr. Equ. Oper. Theory 62, 233-245 (2008)

19. Suárez, D.: The Toeplitz algebra on the Bergman space coincides with its commutator ideal. J. Oper Theory 51(1), 105-114 (2004)

20. Zhu, K.: Operator theory in function spaces. Marcel Dekker, New York (1990)

21. Zhu, K.: Positive Toeplitz operators on weighted Bergman spaces of bounded symmetric domain. J. Oper. Theory $\mathbf{2 0}$ 329-357 (1988)

22. Zhu, K.: Schatten class Toeplitz operators on the weighted Bergman spaces of the unit ball. N. Y. J. Math. 13, 299-316 (2007) 